\title{
Knowledge, attitudes and practices of male circumcision for Human Immunodeficiency Virus (HIV) prevention among voluntary counselling and testing clients in a centre in northern Namibia
}

\author{
Terthu K Ngodji ${ }^{1}$, Jessica Rebert ${ }^{1}$, Ehimario U Igumbor ${ }^{1}$, Vistolina Nuuyoma*2 \\ ${ }^{1}$ School of Public Health, University of the Western Cape, Cape Town, South Africa \\ ${ }^{2}$ School of Nursing, University of Namibia, Keetmanshoop, Namibia
}

Received: October 18, 2016

Accepted: January 19, 2017

Online Published: February 5, 2017

DOI: $10.5430 /$ ijh.v3n1p42

URL: http://dx.doi.org/10.5430/ijh.v3n1p42

\begin{abstract}
Background: There is compelling evidence that male circumcision (MC) is associated with reduced risk of contracting HIV. For this reason, MC is recommended for countries like Namibia where HIV prevalence is high and MC rates are low. Following this recommendation, a national MC campaign was considered to introduce MC as an additional HIV prevention strategy with the intention of rolling it out in communities where MC is not traditionally practised and where heterosexually transmitted HIV infection rates are high. Little is known about the acceptability of $\mathrm{MC}$ in traditionally non-circumcising communities in Northern Namibia.

Objective: This study assessed the knowledge, attitudes and practices of MC among men presenting for voluntary counselling and testing (VCT) in a traditionally non-circumcising ethnic group in the northern Namibia.

Methods: A cross-sectional descriptive study design was used. Semi-structured questionnaires were used to collect data from 331 Oshiwambo-speaking males aged 18 years and older, presenting for VCT services. Data was analysed using Epi-Info 2008, Version 3.5.1.

Results: The self-reported prevalence of MC in the study sample was $15.4 \%$, with $38 \%$ of those reporting being circumcised during their childhood (1-13 years) and 44\% being circumcised for health-related reasons. Most respondents $(\mathrm{n}=241,74.4 \%)$ had heard that MC reduces the men's risk of HIV infection. Up to 53.1\% of the respondents had good knowledge regarding MC; moreover, the majority of the respondents $(n=194,66.6 \%)$ reported that it is easier for uncircumcised men to acquire HIV infection. It was found that men aged 25 to 34 years were more willing to be circumcised than the other age groups.

Conclusions: A high level of knowledge of MC was revealed. Accordingly, the idea of MC is likely to be accepted, especially if it is implemented to reduce the risk of HIV infection.
\end{abstract}

Key Words: Male circumcision, HIV/AIDS, HIV transmission, HIV prevention, Voluntary counselling and testing, Noncircumcising ethnic group, Northern Namibia, Onandjokwe hospital

*Correspondence: Vistolina Nuuyoma, MPH; Email: vistolina.nuuyoma@ gmail.com; Address: P.O. Box 602, Keetmanshoop, Namibia. 


\section{INTRODUCTION}

Sub-Saharan Africa carries the largest burden of the global Human Immunodeficiency Virus/Acquired Immune Deficiency Syndrome (HIV/AIDS) pandemic. In 2008, it was estimated that 33.4 million people were living with HIV worldwide. ${ }^{[1]}$ By the end of 2015, this number had increased to 36.7 million. ${ }^{[2]}$

Namibia is one of the Southern African country heavily affected by the HIV/AIDS epidemic ${ }^{[1]}$ and is one of the top ten countries with the highest prevalence globally. In 2008, the prevalence rate in Namibia was estimated at $17.8 \%$; this increased to $18.8 \%$ in 2010 , but decreased to $18.2 \%$ in 2012 and $16.9 \%$ in $2014 .{ }^{[3]}$ This indicates a decrease in the last two consecutive years. In Namibia, HIV is transmitted primarily by heterosexual transmission. ${ }^{[3]}$

In spite of the rapid spread of HIV, the implementation of various HIV preventive programmes such as HIV voluntary counselling and testing (VCT), condom-use promotion, promotion of abstinence, and treatment programmes for sexually transmitted infections (STIs) has resulted in a remarkable decrease in HIV prevalence and incidence in countries such as Thailand, Uganda and Senegal. ${ }^{[4]}$ However, the rate of HIV infection is increasing in some parts of Africa, for example in Lesotho and some parts of Mozambique. ${ }^{[5]}$

There is well-established evidence that male circumcision (MC) can protect against HIV transmission. Three randomised controlled trials (RCTs) conducted in South Africa, Kenya and Uganda have given strong evidence that safe MC can reduce a male's chance of becoming infected with HIV by approximately $60 \% .^{[6,7]}$ As a result, in March $2007,{ }^{[8]}$ the World Health Organization (WHO) convened an international conference to review the results of the three RCTs and other evidence on MC and HIV prevention. It was then recommended by the WHO and the Joint United Nations Programme on HIV/AIDS (UNAIDS) that "MC be recognized as an additional HIV prevention strategy to reduce the risk of heterosexually acquired HIV infections in countries with low MC prevalence and high HIV prevalence". ${ }^{[8]}$ Since then, interest has been raised by several Southern and East African countries to consider the introduction of MC for HIV prevention. ${ }^{[9]}$ Several modelling studies on the impact of MC on HIV infection in sub-Saharan Africa have shown that MC at $60 \%$ efficacy level could substantially reduce the burden of HIV by reducing the chance of HIV infection in males, especially in Southern Africa where the prevalence of $\mathrm{MC}$ is low and the prevalence of HIV is high. ${ }^{[10,11]} \mathrm{It}$ has been shown that MC alone can significantly reduce HIV infection, although it cannot eliminate the HIV burden in the community. However, Podder ${ }^{[10]}$ concludes that "disease elimination is feasible if $\mathrm{MC}$ is combined with other interventions, such as antiretroviral therapy (ART) and condom use". Fourteen East and Southern African countries, Namibia included, were identified to scale up voluntary MC services to control the spread of HIV epidemic. ${ }^{[8]}$

Therefore, in 2007, the then Namibian Minister of Health and Social Services announced the government's commitment to embark on assessing the possible introduction of safe MC as part of the national strategy for HIV prevention. In 2008, the Namibian government established the MC Task Force to lead the MC scale-up and a policy document was developed. However, little is known about the acceptability of MC intervention programmes among men from traditionally non-circumcising ethnic groups. ${ }^{[12]}$ In particular, it is unclear whether males who have embraced other HIV prevention strategies such as VCT will do the same with MC if implemented as part of a comprehensive HIV prevention programme. According to the Namibia Demographic and Health Survey (DHS) conducted in $2013,{ }^{[13]}$ the national MC prevalence rate is $26 \%$, although this differs across the regions of Namibia. Regions that reported the highest rates are Kunene (51.4\%), Omaheke (48.2\%) and Otjozondjupa (39\%). This could be because most inhabitants of these regions are from traditionally circumcising ethnic tribes. In addition, these regions reported the lowest HIV-prevalence rates from the 2014 National HIV sentinel survey, ranging from $3.9 \%$ to $14.4 \%{ }^{[3]}$ On the other hand, regions such as Ohangwena, Omusati, Oshana, Oshikoto and Zambezi mostly house the traditionally non-circumcising ethnic groups and are reported to have lower MC prevalent rates ranging from $12 \%$ to $18 \% .{ }^{[13]}$ Moreover, these regions reported the highest HIV-prevalence rates ranging from $18 \%$ to $36 \% .^{[3]}$

This study was conducted in 2009 , during the planning and early implementation phase of MC as an HIV-prevention strategy in Namibia. The programme was piloted at the leading state hospitals and was actively scaled up to the regions later. ${ }^{[13]}$

\subsection{Aim}

Our aim was to assess the knowledge, attitudes and practices of MC among men presenting for VCT in a traditionally non-circumcising ethnic group in the Northern Namibia.

\subsection{Objectives}

Our objectives were to:

- measure the MC prevalence among VCT attendees at a testing centre in northern Namibia;

- measure the level of knowledge about MC among male 
VCT attendees;

- describe attitudes of VCT attendees regarding MC.

\section{Material AND Methods}

\subsection{Method}

The researchers chose a quantitative research design to quantify the level of knowledge on and attitudes towards MC, as well as to describe practices related to male circumcision. In addition, a cross-sectional descriptive design using a survey was chosen owing to its suitability for obtaining data on habits, attitudes, beliefs, values and demographics. ${ }^{[14]}$

\subsection{Study setting}

The study was conducted at the Onandjokwe District Hospital, situated in the Oniipa suburb of the Oshikoto region in northern Namibia. The district hospital had a catchment area of 159,621 people in $2007,{ }^{[15]}$ which is $85 \%$ of the estimated 187,500 people living in the Oshikoto region. Over $90 \%$ of the people in the Onandjokwe District speak Oshiwambo as their native language. ${ }^{[16]}$ The Onandjokwe District Hospital runs a special centre, which offers comprehensive HIV services including prevention, treatment and home-based care, well as STI treatment services. The Onandjokwe District Hospital VCT centre has an average of 11 adult male clients for HIV counselling and testing on a daily basis. ${ }^{[17]}$ In addition to the population of Onandjokwe district, the centre also has clients from neighbouring regions such as Ohangwena and Oshana, which are also considered to be traditionally non-circumcising communities. This study was conducted at the VCT centre located on the hospital premises.

\subsection{Population and sampling}

All adult males aged 18 years and older, who belonged to the Oshiwambo ethnic group and visited the Onandjokwe VCT centre for HIV counselling and testing, were eligible for the study. This ethnic group is considered to be one of the noncircumcising groups in Namibia. ${ }^{[13]}$ Clients who were from non-Oshiwambo tribes, as well as those under the age of 18 years, were excluded from the study. Approximately 3,500 adult males attend Onandjokwe VCT centre every year for HIV counselling and testing. ${ }^{[17]}$ Eighty per cent of this male adult population was used to calculate the required sample size. From a total population of 2,800 (80\% of 3,500 clients visiting the VCT centre annually), with a $95 \%$ confidence level, a presumed acceptance rate of MC of $65 \%{ }^{[18]}$ and a $5 \%$ margin of error, a required sample size of 311 study units was calculated using CDC Epi Info 2008 version 3.5.1.

All male clients who visited the Onandjokwe VCT Centre between 28 April 2009 and 6 June 2009 and who met the selection criteria were approached and asked to voluntarily participate in the study, until the required size was reached. This sampling procedure was selected in order to achieve the target sample population with limited resources and time. In addition, this procedure was chosen because a random sampling technique was not possible given that a concise sampling frame of all attendees at the clinic could not be determined prior to data collection. At the same time, the period of data collection (April through June) has no expectation of particular types of patients attending the clinic.

\subsection{Data collection}

An anonymous structured questionnaire with closed-ended questions developed by the investigator was used to collect information from participants. Questions were adapted from previous studies on the acceptability of MC done in South Africa $^{[19]}$ and Kenya. ${ }^{[20]}$ The questions from each of these questionnaires were adjusted and rearranged to accommodate the specific study objective. The questionnaire was designed in English and was translated into Oshiwambo by the investigator and was later revised by an independent native Oshiwambo speaker after the tool had been pre-tested. Participants were introduced to the study while waiting for their HIV test results following HIV pre-test counselling and were requested to voluntarily participate in the study. All those who consented were interviewed individually, using the questionnaire. The interviews, which took 15 to 20 minutes, were conducted in both Oshiwambo and English depending on the participant's preference. To avoid participants being interviewed more than once, participants were asked if they had previously been interviewed before starting the interview.

\subsection{Data analysis}

Questionnaires were checked for completeness and verified by the interviewer before being coded and entered into a MS Access database. The data obtained was analysed using the CDC Epi-Info 2008, version 3.5.1. Descriptive statistics of the demographic characteristics, knowledge, attitudes, beliefs and barriers were presented using absolute numbers, simple percentage, range and measure of central tendency (mean, mode). A chi square test was used to test the association between categorical variables. The level of significance for all statistical tests was set at 0.05 .

\subsection{Validity and reliability}

The sample size was sufficiently large and was drawn from the same ethnic group. The questionnaire was pre-tested prior to the actual data collection on ten participants with similar characteristics to the sample. The questionnaire was administered in languages understood by both the respondents and interviewer. A research assistant was trained to ask 
questions and record the answers in order to ensure standardisation and to avoid interviewer bias. Data quality checks were done in the field as well as before and during the data processing to ensure completeness and consistency.

To address reliability in this study, the same questionnaire was used to collect data from all the participants and only one interviewer conducted all the interviews.

\subsection{Ethical consideration}

The research proposal was granted ethical approval by the University of the Western Cape Research and Ethics Committee. Permission to conduct the research with VCT clients was granted by the Namibian Ministry of Health and Social Services on behalf of the Onandjokwe District Hospital. Participants were given an information sheet explaining the purpose of the study, which was strictly voluntary and participants could withdraw at any time. To ensure confidentiality, no personal identifiers such as names were used on the questionnaire. Participants were assured that data from the study would only be used for research purposes and that it would be kept in a database in a computer and would be password protected.

\section{Results}

\subsection{Description of study participants}

Table 1 presents the demographic characteristics of the respondents in the study. The respondents' ages ranged between 18 and 65 years (mean age 31 years, standard deviation 9.96 , age mode 28 years). The majority ( $n=143,43.2 \%)$, of the respondents were aged between 25 to 34 years. Eightyseven $(26.3 \%)$ of the respondents had no formal or primary education, while $240(72.2 \%)$ had a secondary education or higher. Nearly two-thirds of the respondents $(n=244$, $74.2 \%)$ were unmarried and $86(26 \%)$ were married or cohabitating. Almost all the respondents $(n=324,97.9 \%)$ were Christians. Most of the respondents $(n=244,73.7 \%)$ were unemployed.

\subsection{Male circumcision practices}

Of the 320 respondents who responded to this question, 50 $(15.6 \%)$ reported that they were circumcised, while 270 $(84.4 \%)$ reported that they were not. A total of $19(38 \%)$ respondents had been circumcised during childhood (1-13 years), whereas nine (18\%) had been circumcised during adulthood ( $>20$ years), nine $(18 \%)$ as an infant ( $<1$ year) and seven (14\%) as adolescents (14-19 years).

Among the fifty respondents who had been circumcised, 22 (44\%) circumcisions were performed for health-related reasons, $16(32 \%)$ for traditional/cultural reasons, ten (20\%) for unknown reasons and two (4\%) for other reasons. A total of eight $(36.4 \%)$ respondents who were circumcised for healthrelated reasons and six $(37.5 \%)$ who were circumcised for traditional/cultural reasons were circumcised in childhood (1-13 years). Almost all the respondents $(n=9,90 \%)$, with an unknown reason for being circumcised, had been circumcised below the age of 13 . Of the 50 respondents who had been circumcised, most $(n=31,63.3 \%)$ were circumcised by a doctor at a health facility, $17(30.6 \%)$ were circumcised by a traditional circumciser and two $(6.1 \%)$ did not know who had performed the MC procedure.

Table 1. Socio-demographic characteristics of study participants

\begin{tabular}{lll}
\hline Characteristics & Study sample & Percentage \\
\hline Age & & \\
$18-24$ & 95 & 28.7 \\
$25-4$ & 143 & 43.2 \\
$35-44$ & 54 & 16.3 \\
$45-54$ & 26 & 7.9 \\
$55+$ & 13 & 3.9 \\
Marital status & & \\
Unmarried & 244 & 73.7 \\
Married/cohabitating & 86 & 26 \\
Not specified & 1 & 0.3 \\
Level of education & & \\
Primary and lower & 87 & 26.3 \\
Secondary and higher & 239 & 72.2 \\
Not specified & 5 & 1.5 \\
Employment & & \\
Employed & 85 & 25.3 \\
Unemployed & 244 & 73.7 \\
Not specified & 2 & 0.6 \\
Religion & & \\
Christian & 324 & 97.9 \\
Non-Christian & 5 & 1.5 \\
Unspecified & 2 & 0.6 \\
\hline
\end{tabular}

The most common complications of MC reported were infection $(n=16,45.7 \%)$, followed by bleeding $(n=5,14.3 \%)$. Other complications reported include tissue loss (reduced size of tip of penis) $(n=3,8.6 \%)$; urinal retention $(n=1$, $2.9 \%)$; incomplete circumcision $(\mathrm{n}=1,2.9 \%)$; and the remainder $(n=9,25 \%)$ was other complications (see Table 2).

In order to identify whether there were socio-demographic differences between circumcised and uncircumcised men, the study assessed whether the demographic characteristics differed according to the MC status of the respondents in the study. Age, employment, marital status, level of education and religion were each compared by circumcision status and the differences were not statistically significant. 
Table 2. Complications of MC

\begin{tabular}{|c|c|}
\hline Male circumcision complications & $\begin{array}{l}\mathrm{N}=35 \\
\text { Frequency (\%) }\end{array}$ \\
\hline Infections & $16(45.7)$ \\
\hline Tissue loss & $3(8.6)$ \\
\hline Urinary retention & $1(2.9)$ \\
\hline Incomplete circumcision & $1(2.9)$ \\
\hline Bleeding & $5(14.3)$ \\
\hline Other complications & $9(25.7)$ \\
\hline
\end{tabular}

\subsection{Knowledge of male circumcision as a HIV preven- tion strategy}

Risk-related knowledge of respondents about MC, HIV/AIDS and STIs was determined by the respondents' general knowledge of MC and its risk associations with HIV infection and STIs. Excluding the seven participants who did not respond to the knowledge questions, most respondents $(\mathrm{n}=241,74.4 \%)$ had heard that MC reduces men's risk of HIV infection. The majority $(n=214,66 \%)$ had also heard that MC reduces men's risk of STIs. When age, marital status, religion, educational level and employment status were compared with the level of knowledge, people who were employed were found to be likely to have a good knowledge of MC and related health aspects $(p<.003)$.

\subsection{Attitudes to circumcision}

Table 3 indicates the risk-related attitudes of participants towards male circumcision. The analysis excludes the eleven participants who did not report their circumcision status, thus the numerator is 320 . The results indicated that 194 out of the $320(60.7 \%)$ respondents (37 circumcised and 157 uncircumcised) reported that it is easier for uncircumcised men to acquire HIV infection. A high proportion of uncircumcised respondents, 155 out of 270 (57.4\%), and the majority of circumcised respondents, 35 out of 37 (70\%), believed that it is easier for uncircumcised men to acquire STIs. Only 143 out of $320(44.7 \%)$ of the respondents (54\% circumcised and $43 \%$ uncircumcised) believed than it is easier for uncircumcised men to get penile cancer. However, uncircumcised respondents $(24.1 \%)$ were much more likely than circumcised respondents $(6 \%)$ to report that there is no relationship between circumcision status and risk of penile cancer $(p=$ .0335). Furthermore, a higher proportion of the respondents, 267 out of $320(83.4 \%)$, reported that it is easier for a circumcised man to maintain good penile hygiene. Circumcised and uncircumcised respondents did not differ in their beliefs about the relationship between circumcision status and maintaining penile hygiene ( $84 \%$ versus. $83.3 \%, p=.5339$ ).

Table 3. Risk-related attitudes about male circumcision

\begin{tabular}{|c|c|c|c|}
\hline Attitudes & $\begin{array}{l}\text { Circumcised } \\
\mathbf{N}=\mathbf{5 0} \\
\text { n (\%) }\end{array}$ & $\begin{array}{l}\text { Uncircumcised } \\
\mathrm{N}=\mathbf{2 7 0} \\
\mathbf{n}(\%)\end{array}$ & $\begin{array}{l}\text { Total } \\
N=320 \\
\text { n (\%) }\end{array}$ \\
\hline \multicolumn{4}{|c|}{ Easier to get HIV when } \\
\hline Circumcised & $0(0)$ & $10(3.7)$ & $10(3.1)$ \\
\hline Uncircumcised & $37(74)$ & $157(58.1)$ & $194(60.7)$ \\
\hline No difference & $12(24)$ & $83(30.7)$ & 95 (29.4) \\
\hline Do not know & $1(2)$ & $20(7.4)$ & $21(6.6)$ \\
\hline \multicolumn{4}{|c|}{ Easier to get other STIs when } \\
\hline Circumcised & $2(4)$ & $11(4.1)$ & $13(4.1)$ \\
\hline Uncircumcised & $35(70)$ & 155 (57.4) & $190(59.4)$ \\
\hline No difference & $11(22)$ & 79 (29.3) & $90(28.1)$ \\
\hline Do not know & $2(4)$ & $25(9.3)$ & $27(8.4)$ \\
\hline \multicolumn{4}{|c|}{ Easier to keep penile hygiene when } \\
\hline Circumcised & $42(84)$ & $225(83.3)$ & $267(83.4)$ \\
\hline Uncircumcised & $0(0)$ & $7(2.6)$ & $7(2.2)$ \\
\hline No difference & $6(12)$ & $33(12.2)$ & $39(12.2)$ \\
\hline Do not know & $2(4)$ & $5(1.9)$ & $7(2.2)$ \\
\hline
\end{tabular}

Two hundred (60.4\%) respondents disagreed that circumcised men have more sexual feelings than uncircumcised men. Only 134 (39\%) respondents agreed that circumcised men enjoy sex more than uncircumcised men, and 139 (42\%) respondents agreed that women prefer men who are circumcised. Almost all the respondents $(\mathrm{n}=289,87.3 \%)$ disagreed that circumcised men can safely have sex without using a condom and do not get infected with HIV. Two-thirds of the respondents, 261 (78.9\%) disagreed that MC proves manhood, and $264(74 \%)$ respondents agreed that it is very important for all males to be circumcised irrespective of their ages, while $79.8 \%$ disagreed that MC is an old practice in 
their community and does not need to be reintroduced.

Of the 270 uncircumcised respondents, 204 (75.5\%) reported that they would like to be safely circumcised if circumcision reduced the chance of HIV infections and if costs were low. When compared, men were more willing to be circumcised if they were 25 to 34 years $(83.9 \%, p<.001)$, unmarried $(82.1 \%, p<.001)$ and had a secondary education level or higher $(79.8 \%, p<.011)$. No other socio-demographic characteristics were found to be statistically associated with uncircumcised men's willingness to be circumcised. Moreover, almost all the respondents $(n=302,94.4 \%)$ preferred their sons or any young males they knew to be circumcised. Respondents who were circumcised indicated a higher level of preference than uncircumcised respondents (98\% vs $93.7 \%$, $p<.05)$. The majority of respondents $(\mathrm{n}=132,39.9 \%)$ preferred "less than 1 year" as the ideal age of performing $\mathrm{MC}$, while $89(26.9 \%)$ preferred " $1-13$ years". Only $11(3.3 \%)$, respondents preferred adulthood ( $>20$ years) as the ideal time for performing MC.

Three hundred and four respondents $(91.8 \%)$ preferred MC to be performed at a health facility by medical staff. Only one $(0.9 \%)$ respondent preferred $\mathrm{MC}$ to be performed outside the health facility by a traditional circumciser, while 16 $(4.8 \%)$ had no preferences.

\section{Discussion}

In this study, we measured prevalence and knowledge of $\mathrm{MC}$ and described the attitudes to MC among VCT clients from a non-circumcising ethnic group. The study revealed an MC prevalence rate of $15.6 \%$, which is lower than the Namibian rate, which was estimated to be $26 \%$ in men of aged 15 to 49. ${ }^{\text {[21] }}$ Despite the rollout of a male circumcision programme in Namibia, the Oshikoto region where the Onandjokwe district is located reported an MC prevalence of just $15.5 \%$ in 2013. ${ }^{21]}$ This is an indication that MC is not commonly practised in the area, which is dominated by the Oshiwambo tribes. Although it is said that MC was practised among Oshiwambo tribes as part of initiation rituals over 100 years ago, ${ }^{[22]}$ its discontinuation is evident in the low prevalence. Social developments such as the development of combined kingship and the introduction of Christianity by the arrival of Europeans in Namibia may have contributed to the discontinuation of the practice among Oshiwambo-speaking people. ${ }^{[22,23]}$ The results of the current study revealed that circumcision is performed at young ages (below 13 years) among the study population and mostly for health-related reasons. This result contradicts reports by Salokoski ${ }^{[22]}$ that MC in the Oshiwambo tribe is said to have been practised only on adult men for initiation from childhood into adulthood. This may partially suggest a gradual shift in MC practices

Published by Sciedu Press from traditional to health-related practice in this study group. No literature was found to establish the actual reason for circumcision practice among the general population of the Oshiwambo tribes.

The findings of this study are very similar to the prevalence rate of MC in the Mutare rural district in Zimbabwe, which revealed a rate of $17 \% .{ }^{[24]}$ Similar to the Onandjokwe district, the Mutare community is also considered to be noncircumcising. The findings from studies in traditionally uncircumcised societies in South Africa and Zimbabwe show no significant differences with regard to circumcision status and employment, relationship status, level of education or religion. ${ }^{[25]}$ Such findings concur with those of this study.

The results of the study confirmed the good knowledge of MC among the study group, in spite of the fact that is not commonly practised. Although the majority (85\%) of the participants were not circumcised, almost all the participants $(98 \%)$ were aware of MC. These results are similar to those of studies done in non-circumcising communities in South Africa, where was found that $93.3 \%$ had heard about circumcision, although only $23.3 \%$ of respondents were circumcised. ${ }^{[26]}$ The awareness of MC in this study may be attributed to the announcement of the South Africa, Kenya and Uganda RCTs on the association between MC and HIV infection $^{[6,7]}$ and the announcement by the Namibian government to embark on the introduction of safe MC to the public in 2008. The association between MC and health benefits (reduced risk of HIV and STIs, penile hygiene enhancement) was prevalent in this study. Despite residing in an area where circumcision is little practised and despite being uncircumcised, the study revealed that a high proportion of men associate circumcision with a reduced risk of acquiring STIs and HIV. These results concur with findings from non-circumcising communities where participants believe that MC improves penile hygiene and offers protection against diseases like HIV/AIDS. ${ }^{[25]}$ Furthermore, circumcised men in this study expressed more positive beliefs about circumcision and its health benefits than uncircumcised men. Compared to $58.1 \%$ of uncircumcised men, $74 \%$ of circumcised men reported that it is easier to acquire HIV infection if the male is uncircumcised. Although a higher proportion of participating men expressed a belief that circumcision reduces the chance of getting HIV infections, $7.6 \%$ of the 331 respondents agreed that circumcised men can safely have sex without using a condom and do not get infected with HIV. These results suggest a perception of false HIV safety, as some people may believe that MC provides complete protection.

Therefore, MC intervention programmes must stress that 
circumcision does not provide complete protection against HIV/STIs and should not be considered a substitute for other prevention methods, but merely part of an HIV prevention programme.

The results gave an indication that participants believed that circumcision influences sexual performance for men themselves as well as for their female partner. About 39.6\% of the respondents agreed that circumcised men enjoy sex more than uncircumcised men, while $42 \%$ of the respondents agreed that women prefer men who are circumcised. However, the study could not establish if those who were circumcised had undergone the procedure for the purpose of attaining increased sexual pleasure. In Kenya, more than half of the uncircumcised men $(55 \%)$ believed that women enjoy sex more with circumcised men, and this belief is a strong predictor of preference for circumcision. ${ }^{[20]}$ Therefore, it is worthwhile noting the conclusion that an MC promotion campaign within this study population might have more impact if it were to promote "better sex" over "safer sex". ${ }^{[19]}$ It is, however, unlikely that men in Onandjokwe district may decide to undergo the procedure because of the belief that MC enhances sexual pleasure.

Although $84.4 \%$ of men in the study population were uncircumcised at the time of the study, $75.6 \%$ of respondents indicated after being informed of the risks and benefits of male circumcision that they would like to be circumcised safely if the procedure lowered the risk of HIV and the cost was low. Surprisingly, acceptability among men in the 45 years and above age group was very low. They were the least likely to be willing to be circumcised, with only $32.1 \%$ indicating that they would like to undergo $\mathrm{MC}$ if it were offered to them. This result suggests that if $\mathrm{MC}$ were implemented in the 18 to 45 age group, it would be more likely to be accepted.

Because MC is less common in the Onandjokwe district community, some men held the belief that it is against their tradition to circumcise. This is similar to the results of Pappas-DeLuca et al., ${ }^{[27]}$ which found that some Oshiwambospeaking respondents expressed a concern that being circumcised amounted to adopting the culture of other societies, thus preventing them from taking the procedure.

\section{Recommendation}

From this study, the following recommendations are made:
A behaviour change communication campaign for both women and men would help strengthen the facilitators of $\mathrm{MC}$ and address the perceived barriers to MC. This can be done through community discussion approaches aimed at changing some of the negative perceptions mentioned in this study. There is a need to design an Information, Education and Communication (IEC) strategy to appropriately promote MC as an HIV-prevention strategy, with proper explanation that $\mathrm{MC}$ is not a complete protective intervention for HIV transmission. The IEC should address the role of MC as an additional measure to several HIV-prevention strategies, which include the practice of safer sex (use of condom, being faithful and sexual abstinence). Information on MC and HIV should be made available, accessible and acceptable to the public with a focus on males and their partners. It is also important to improve and initiate training for medical and traditional circumcisers as well as the information that is available to all males and their partners.

\section{Conclusions}

This study aimed to assess the knowledge, attitudes and practices of MC as an HIV-prevention intervention among adult males attending VCT at the Onandjokwe District Hospital. A high level of knowledge of MC was found among participants, particularly its potential to reduce the risk of HIV infection and STIs and enhance penile hygiene. The majority of the respondents reported that it is easier for uncircumcised men to acquire HIV infection and STIs. MC would probably be accepted in this study area especially if implemented to reduce the risk of $\mathrm{HIV}$ infection. It was also found that almost all of the respondents would want MC to be performed in a hospital. This study finding would be useful to health policy makers in the design of community health education programmes for MC implementation in terms of its adoption as an HIV-prevention strategy in Namibia.

\section{ACKNOWLEDGEMENTS}

We thank the participants who willingly contributed to the study. The authors contributed as follows: T. K. N. is the principal investigator, J. R. and E. U. E. supervised the project, and V. N. wrote the manuscript.

\section{CONFlicts OF INTEREST Disclosure}

The authors declare no conflicts of interest. 


\section{REFERENCES}

[1] Joint United Nations Programme on HIV/AIDS (UNAIDS) and World Health Organization (WHO). 2009. Available from: http://data.unaids.org/pub/Report/2009/JC1700_Epi _Update_2009_en.pdf

[2] World Health Organization (WHO). Global Health Observatory Data: HIV/AIDS. 2016. Available from: http: //www . who.int/gho/hi $\mathrm{v} / \mathrm{en} /$

[3] Ministry of Health and Social Services (MoHSS). Surveillance Report of the 2014 National HIV Sentinel Survey. 2014. Available from: http://www.mhss.gov.na/files/downloads/12f_ $2014 \% 20$ National $\% 20$ HIV $\% 20$ Sentinel $\% 20$ Survey .pdf

[4] Wegbreit J, Bertozzi S, De Maria LM, et al. Effectiveness of HIV Prevention Strategies in Resource-Poor Countries: Tailoring the Intervention to the Context. AIDS, 2016; 20(9): 1217-35. PMid: 16816550. Available from: https://siid.insp.mx/documentos/curric ulo/articulos/com-1875439.pdf

[5] Joint United Nations Programme on HIV/AIDS (UNAIDS) and World Health Organization (WHO). 2008. Report on the Global AIDS Epidemic. Available from: http://www . unaids.org/sites/default/files/media _asset/jc1510_2008globalreport_en_0.pdf

[6] Auvert B, Taljaard D, Lagarde E, et al. Randomized Controlled Intervention Trial of Male Circumcision for Reduction of HIV Infection Risk: The ANRS 1265 Trial. PLoS Medicine. 2005; 2(11): 1112-22. https://doi.org/10.1371/journal.pmed.0020298

[7] Bailey RC, Moses S, Parker CB, et al. Male Circumcision for HIV Prevention in Young Men, Kenya: A Randomized Controlled Trial. Lancet. 2007; 369(9562): 643-56. https ://doi.org/10.1016/ S0140-6736(07)60312-2

[8] World Health Organization and Joint United Nationals Programme on HIV/AIDS [WHO/UNAIDS]. New Data on Male Circumcision and HIV Prevention: Policyand Programme Implications Conclusion and Recommendations. WHO/UNAIDS Technical Consultation, Montreux. 2007. Available from: http://www . unaids.org/sites/default/files/media_a sset/mc_recommendations_en_0.pdf

[9] Joint United Nations Programme on HIV/AIDS. Using the DecisionMakers" Programme Planning Tool for Male Circumcision Service Scale-up. Meeting Report, Dakar, Sénégal. 2008. Available from: http://data.unaids.org/pub/Report/2009/jc1699 _dakar_meeting_report_en.pdf

[10] Podder CN, Sharomi O, Gumel AB, et al. To Cut or Not to Cut: A Modeling Approach for Assessing the Role of Male Circumcision in HIV Control. Bulletin of Mathematical Biology. 2007; 69(1): 2447-66. https://doi.org/10.1007/s11538-007-9226-9

[11] Williams BG, Lloyd-Smith JO, Gouws E, et al. The Potential Impact of Male Circumcision on HIV in Sub-Saharan Africa. PloS Medicine. 2006; 3(7): e262-62. https://doi.org/10.1371/journal.pm ed. 0030262

[12] Katuta F. Country Experiences in the Scale-Up of Male Circumcision in the Eastern and Southern Africa Region: Two Years and Counting. 2009. A Sub-Regional Consultation. Windhoek.

[13] Ministry of Health and Social Services. Namibia Demographic and Health Survey 2013. Available from: https://dhsprogram.com /pubs/pdf/FR298/FR298.pdf
[14] Maree K, editor. First Steps in Research. Pretoria: Van Schaik; 2016.

[15] Onandjokwe District Hospital. Onandjokwe District Population Estimate Based on 2001 Population Census. Onandjokwe Hospital, Namibia. 2008.

[16] Health Communication Partnership. HIV/AIDS Lifestyles, Knowledge, Attitudes and Practices: A Baseline Household Survey of Residents from Onandjokwe. 2004. Available from: http://www.hcpartnership.org/Programs/Africa/nami bia/onandjokwe_household_baseline.pdf

[17] Onandjokwe District Hospital. Voluntarily Counseling and Testing Records. Onandjokwe Hospital, Namibia. 2008.

[18] Westercamp N, Bailey RC. Acceptability of Male Circumcision for Prevention of HIV/AIDS in Sub-Saharan Africa: A Review. AIDS Behavior. 2007; 11(3): 341-55. http://dx.doi.org/10.1007/s 10461-006-9169-4

[19] Scott BE, Weiss HA, Viljoen JI. The Acceptability of Male Circumcision as an HIV Intervention among a Rural Zulu population in KwaZulu-Natal South Africa. AIDS Care. 2005; 17(3): 304-13. https://doi.org/10.1080/09540120412331299744

[20] Mattson CL, Bailey RC, Muga R, et al. Acceptability of Male Circumcision and Predictors of Circumcision preference among Men and Women in Nyanza Province in Kenya. AIDS Care. 2005; 17(2): 182-94. https://doi.org/10.1080/09540120412331299744

[21] Ministry of Health and Social Service (MoHSS) and Namibia Statistic Agency (NSA). Namibia Demographic and Health Survey. 2013 Available from: https://dhsprogram.com/pubs/pdf/FR298/ FR298.pdf

[22] Salokoski M. How Kings are Made - How Kingship Changes: A study of Rituals and Ritual Change in Pre-Colonial and Colonial Owamboland, Namibia. Unpublished dissertation. 2006. Available from: http://ethesis.helsinki.fi/julkaisut/val/sosi o/vk/salokoski/howkings.pdf

[23] Iipinge E, Shitundeni J. Initiation Rites in Kavango. Windhoek: Social Sciences Division of the Multi-Disciplinary Research Centre of the University of Namibia. 1999.

[24] Chiringa IO, Ramathuba DU, Mashau NS. Factors contributing to the low uptake of medical male circumcision in Mutare Rural District, Zimbabwe. Afr J Prm Health Care Fam Med. 2016; 8(2): a966. https://doi.org/10.4102/phcfm.v8i2.966

[25] Khumalo-Sakutukwaa G, Lanea T, Van Rooyen BH, et al. Understanding and addressing socio-cultural barriers to medical male circumcision in traditionally non-circumcising rural communities in sub-Saharan Africa. Culture, Health \& Sexuality. 2013; 15(9): 1085100. https ://doi.org/10.1080/13691058.2013.807519

[26] Hoffman JR, Arendse KD, Larbi C, et al. Perceptions and knowledge of voluntary medical male circumcision for HIV prevention in traditionally non-circumcising communities in South Africa. Global Public Health. 2015; 10(5-6): 692-707. https://doi.org/10.1 $080 / 17441692.2015 .1014825$

[27] Pappas-DeLuca KA, Simeon F, Kustaa F. Preliminary Results of the Report on Findings from Qualitative Research on Male Circumcision in Namibia: Unpublished report. 2008. Windhoek: Ministry of Health and Social Services. 